\title{
Actualización en Presentación y Patogénesis de la Displasia Broncopulmonar
}

\author{
ALDO BANCALARI M. ${ }^{1}$ \\ 1. Profesor Asociado de Pediatría. Director Programa de Neonatología, Departamento de Pediatría, Facultad de Medicina, \\ Universidad de Concepción.
}

\begin{abstract}
Update on Presentation and Pathogenesis of Brochopulmonary Dysplasia
\end{abstract}

Bronchopulmonary dysplasia (BPD) remains as the most frequent chronic lung disease seen among babies with very low birth weight, contributing to their morbidity and mortality. An increase in the survival of very immature babies due to improvement in pre and post natal care, has resulted in an increase in the number of newborns with BDP, although there have been no changes in the actual incidence of the disease. Objective: To describe the evolution of DBP in recent decades, the current definition, and to describe and analyze the risk factors involved in the pathogenesis of this disease. Until a few years ago, the terms BPD and chronic lung disease were used as synonyms. After the workshop sponsored by the National Institute of Health in the United States in 2001, it was recommended that the term BPD be used to describe the pulmonary sequelae of immature babies. Classic severe BPD, as described by Northway et al over forty years ago, has evolved into milder forms of chronic pulmonary damage, the so-called "new BPD", characterized by impairment of alveolarización and vascularization of the immature lung in response to multiple injuries. BPD is a multifactorial disease where major risk factors are related to pulmonary immaturity, hyperoxia, baro/volutrauma, as well as inflamation and infection. Genetic susceptibility has recently been shown to be another important risk factor. Conclusion: Bronchopulmonary Dysplasia continues to be the most frequent sequelae affecting low birth weight infants. In the past four decades, the disease has been better defined, and new pathogenetic risk factors have been established.

(Key words: Bronchopulmonary dysplasia, prematurity, oxygen therapy, mechanical ventilation, ductus arteriosus).

Rev Chil Pediatr 2009; 80 (3): 213-224

\section{RESUMEN}

La Displasia Broncopulmonar (DBP) continúa siendo la enfermedad pulmonar crónica más frecuente que afecta al recién nacido de muy bajo peso, contribuyendo a su morbilidad y mortalidad. El aumento en la sobrevida de los recién nacidos muy inmaduros, debido a la mejoría en el cuidado pre y post natal, ha aumentado el número de recién nacidos con displasia, sin cambios en su incidencia. El objetivo de esta revisión es representar los cambios en la presentación clínica de la DBP en las últimas décadas y describir

Trabajo recibido el 24 de noviembre de 2008, devuelto para corregir el 13 de enero de 2009, segunda versión el 21 de enero de 2009, aceptado para publicación el 23 de marzo de 2009.

Correspondencia a:

Dr. Aldo Bancalari M.

E-mail: abancalari@entelchile.net 
la definición recientemente instituida, junto con analizar y actualizar los factores de riesgos involucrados en la patogénesis de esta enfermedad. Hasta hace algunos años el término DBP o Enfermedad Pulmonar Crónica se usaban como sinónimos; sin embargo luego del taller patrocinado por Instituto Nacional de Salud de Estados Unidos en el año 2001, se recomendó utilizar el término de DBP para describir las secuelas pulmonares del recién nacido muy inmaduro. La DBP clásica severa descrita por Northway y cols hace más de 40 años, ha evolucionado a formas más leves de daño pulmonar crónico, la denominada "Nueva DBP", caracterizada por un deterioro y/o detención de la alveolarización y vascularización del pulmón inmaduro en respuesta a múltiples injurias. La DBP es una enfermedad multifactorial siendo los principales factores de riesgo la inmadurez pulmonar, la hiperoxia, el baro-volutrauma, y la inflamación-infección. Recientemente se ha demostrado que la susceptibilidad genética puede ser otro factor de riesgo. La ventilación mecánica continúa siendo un importante factor de riesgo, por lo cual debe ser utilizada con precaución y sólo cuando esté claramente indicada. La persistencia del ductus arterioso se ha asociado también a DBP, por lo cual el cierre farmacológico precoz podría disminuir la incidencia de esta complicación. Conclusión: La DBP continúa siendo la secuela pulmonar crónica más frecuente que afecta al RN de muy bajo peso. En las últimas 4 décadas se ha podido definir mejor esta enfermedad y se han establecido nuevos factores de riesgos involucrados en su patogénesis.

(Palabras clave: Displasia broncopulmonar, prematuridad, oxígenoterapia, ventilación mecánica, ductus arterioso persistente).

Rev Chil Pediatr 2009; 80 (3): 213-224

\section{Introducción}

La Displasia Broncopulmonar (DBP) es la enfermedad pulmonar crónica que más frecuentemente ocurre en los recién nacidos prematuros de muy bajo peso, que han necesitado ventilación mecánica y oxígeno suplementario por un distrés respiratorio. Esta enfermedad fue descrita por Northway y cols ${ }^{1}$, hace más de 40 años en un grupo de $32 \mathrm{RN}$ de pretérmino con peso mayor de $1500 \mathrm{~g}$ al nacer, que presentaron dificultad respiratoria severa y recibieron una ventilación mecánica prolongada y agresiva con presiones inspiratorias elevadas y altas concentraciones de oxígeno (mayor de $80 \%$ ). Estos parámetros ventilatorios produjeron un daño pulmonar crónico caracterizado por requerimientos prolongados de oxígeno y diversas alteraciones radiológicas ${ }^{1}$. Se describieron 4 etapas que terminaban en daño pulmonar severo, con falla respiratoria, caracterizada por hipoxemia e hipercapnia, acompañado con frecuencia de cor pulmonale. Los cambios radiológicos en la etapa más avanzada mostraban áreas de colapso, con aumento de la densidad pulmonar, acompañada de hiperinsuflación, especialmente de los lóbulos inferiores e imágenes cordonales de fibrosis ${ }^{1}$.

Sin embargo, en las últimas dos décadas la
DBP clásica descrita anteriormente ha sido reemplazada mayoritariamente por formas más leves de injuria pulmonar crónica, la denominada "Nueva DBP"2. Esta presentación de la DBP tiene un mejor pronóstico y menos secuelas a largo plazo, pero su incidencia no ha disminuido ${ }^{3,4}$. Este cambio se debería al avance en el cuidado perinatal de los últimos años, dado por la administración de corticoides prenatales en parto prematuro que aceleran la maduración pulmonar fetal, el uso precoz de surfactante en RN con distrés respiratorio, estrategias ventilatorias más conservadoras, nuevos modos ventilatorios, manejo más agresivo del ductus arterioso persistente, una nutrición parenteral más precoz, además de otras intervenciones ${ }^{2-4}$. Estos avances perinatales han significado un aumento en la sobrevida de los RN de extremo bajo peso $(<1000 \mathrm{~g})$ lo que explicaría en parte la mantención de la incidencia de la DBP, por afectar esta patología principalmente a los $\mathrm{RN}$ muy inmaduros ${ }^{3,4}$.

Esta evolución en la presentación de la DBP, se ha debido también al hallazgo de nuevos factores de riesgo, que intervienen en la patogénesis de esta enfermedad, como son las infecciones, las deficiencias de algunos nutrientes y antioxidantes, además de factores genéticos últimamente descritos ${ }^{2,4}$. 
Recientemente también se han introducido cambios en la definición de la DBP, con el objeto de uniformar criterios y poder predecir mejor el pronóstico de esta patología ${ }^{5}$. Paralelamente en los últimos años han aparecido nuevas estrategias de prevención, algunas de las cuales están en desarrollo y otras en etapa de investigación, con la finalidad de disminuir la incidencia y severidad de la DBP.

El objetivo de la presente revisión, es representar la evolución experimentada por la DBP en las últimas décadas, describir la última definición instituida y analizar los factores de riesgo involucrados en la patogénesis de esta enfermedad.

\section{Definición de DBP}

Desde la descripción inicial de Northway ${ }^{1}$ la historia natural de esta afección ha cambiado, y nuevas definiciones han sido propuestas. En 1979 Bancalari y cols ${ }^{6}$, definen como DBP al cuadro de insuficiencia respiratoria neonatal prolongada en un neonato que habiendo necesitado ventilación mecánica por algunos días inicialmente, persiste con requerimientos de oxígeno y alteraciones radiológicas a los 28 días de vida. Sin embargo, con el aumento de la sobrevivencia de los RN más inmaduros, diversos autores notaron que las alteraciones radiológicas diferían a las previamente descritas, cuestionándose la importancia de los requerimientos de oxígeno durante los primeros 28 días de vida.

En 1988 Shennan y cols ${ }^{7}$, introducen el concepto de dependencia de oxígeno a las 36 semanas de edad postconcepcional, como definición de DBP, teniendo esta definición una relación más estrecha con su pronóstico. Este diagnóstico clínico de DBP a las 36 semanas de edad postconcepcional se ha asociado a futuras alteraciones pulmonares o neurosensitivas ${ }^{8,9}$. No obstante con esta clasificación los RN mayores de 32 semanas de edad gestacional no quedaban bien clasificados; por lo cual el Instituto Nacional de Salud de EE.UU. efectuó en el año 2001 un taller de DBP. Luego de este taller se publicó el consenso de la nueva definición de DBP, en que se dividen los RN en menor de 32 semanas y mayor o igual a 32 semanas de edad gestacional, clasificando la DBP en leve, moderada o severa según los requerimientos de oxígeno y el apoyo ventilatorio $^{9}$ (tabla 1).

Recientemente Walsh y cols ${ }^{10}$, introdujeron el término de definición fisiológica de la DBP. Al aplicar esta definición fisiológica en un estudio prospectivo en 17 centros americanos la incidencia promedio de DBP se redujo en un $10 \%$ (35\% a $25 \%)$. Esta definición se basa en reducir el oxígeno en aquellos recién nacidos con diagnóstico de DBP a las 36 semanas de edad postconcepcional que requieren $30 \%$ de oxígeno o menos, y mantenerlos por un período de 30 minutos respirando aire ambiental con

Tabla 1. Definición de displasia broncopulmonar. Criterios diagnósticos (NICHD/NHLBI/ORD WORSHOP, 2000)

\begin{tabular}{|c|c|c|}
\hline Edad gestacional & $<32$ semanas & $\geq 32$ semanas \\
\hline Edad al diagnóstico & $\begin{array}{l}36 \text { semanas edad corregida o alta domiciliaria, } \\
\text { lo que se cumpla primero }\end{array}$ & $\begin{array}{l}>28 \text { días, pero < } 56 \text { días de vida o alta } \\
\text { domiciliaria, lo que se cumpla primero }\end{array}$ \\
\hline \multicolumn{3}{|c|}{ Tratamiento con $\mathrm{O}_{2}>21 \%$ por más de 28 días } \\
\hline DBP leve & $\begin{array}{l}\text { Respirando aire ambiental a las } 36 \text { semanas edad } \\
\text { corregida o al alta, lo que se cumpla primero }\end{array}$ & $\begin{array}{l}\text { Respirando aire ambiental a los } 56 \text { días de vida o } \\
\text { al alta, lo que se cumpla primero }\end{array}$ \\
\hline DBP moderada & $\begin{array}{l}\text { Necesidad de oxígeno }<30 \% \text { a las } 36 \text { semanas } \\
\text { de edad corregida o al alta, lo que se cumpla } \\
\text { primero }\end{array}$ & $\begin{array}{l}\text { Necesidad de oxígeno }<30 \% \text { a los } 56 \text { días de } \\
\text { vida o al alta, lo que se cumpla primero }\end{array}$ \\
\hline DBP severa & $\begin{array}{l}\text { Necesidad de oxígeno } \geq 30 \% \text { y/o presión positiva } \\
\text { (CPAP o VM) a las } 36 \text { semanas edad corregida o } \\
\text { al alta, lo que se cumpla primero }\end{array}$ & $\begin{array}{l}\text { Necesidad de oxígeno } \geq 30 \% \text { y/o presión positiva } \\
\text { (CPAP o VM) a los } 56 \text { días de vida o al alta, lo } \\
\text { que se cumpla primero }\end{array}$ \\
\hline
\end{tabular}


control de saturometría y frecuencia cardíaca permanente. Aquellos niños que mantenían durante esa media hora de análisis, saturometrías sobre $90 \%$, se consideraban que no presentaban $\mathrm{DBP}^{10}$. Esta definición fisiológica también significó minimizar las variaciones en el diagnóstico de DBP entre los diferentes centros evaluados ${ }^{10}$. La ventaja de esta definición estaría en que todos los niños se evaluarían en forma similar, independiente de las diferentes prácticas clínicas que ocurren en los diversos centros neonatales.

\section{Incidencia}

La incidencia de la DBP en los RN prematuros es inversamente proporcional a la edad gestacional y al peso de nacimiento ${ }^{3,4,11}$. La incidencia de esta afección reportada es muy variable entre los diferentes centros por diversas razones como; definición utilizadas, diferentes poblaciones analizadas, diferencias en el manejo pre y post natal entre los centros, sobrevivencia de los prematuros etc ${ }^{3,4,11}$. En EE.UU. en prematuros con peso de nacimiento entre 500 y $1500 \mathrm{~g}$ se han publicado incidencias tan variables que oscilan entre un $3 \mathrm{y}$ $43 \%{ }^{12}$; y en 16 unidades de Sudamérica en RN de muy bajo peso, la incidencia de DBP varió entre 8,6 y $44,6 \%{ }^{11}$. Se ha reportado que aproximadamente el $75 \%$ de los niños afectados de DBP pesaron menos de $1000 \mathrm{~g}$ al nacer, y la incidencia puede llegar hasta un $85 \%$ en $\mathrm{RN}$ con peso de nacimiento entre 500 y $699 \mathrm{~g}^{13}$. En un estudio reciente donde la DBP fue definida como dependencia de oxígeno a las 36 semanas postconcepcionales la incidencia fue de $52 \%$ en los niños con peso de nacimiento entre 500 y $750 \mathrm{~g} ; 34 \%$ en niños con peso entre 751 y $1000 \mathrm{~g}$; y sólo un 7\% en niños con peso de nacimiento entre 1201 y $1500 \mathrm{~g}^{14}$.

En el Hospital Gmo. Grant Benavente de Concepción, Chile, entre los años 2000-2007 la incidencia global de DBP en los RN de muy bajo peso sobreviviente a los 28 días de vida fue de $27,2 \%$ y a las 36 semanas postconcepcionales $15,4 \%$; siendo mayor, mientras menor es el peso de nacimiento del $\mathrm{RN}$, tal como ocurre en la mayoría de las series clínicas (tabla 2).

\section{Cuadro clínico}

Actualmente se pueden distinguir 2 presentaciones: La DBP Clásica y la Nueva DBP.

La DBP Clásica es aquella que se presenta en $\mathrm{RN}$ prematuros sobrevivientes a un distrés respiratorio severo que han requerido ventilación mecánica con parámetros elevados: altas concentraciones de oxígeno y de presiones inspiratorias, con persistencia de síntomas y signos de dificultad respiratoria y dependencia de oxígeno adicional por períodos prolongados; junto con alteraciones radiológicas crónicas, entre las cuales se pueden observar, imágenes quísticas con hiperinsuflación pulmonar y líneas intersticiales que corresponden a fibrosis pulmonar (figura 1). En estos niños se puede observar daño de la vía aérea, hipertrofia de glándulas mucosas y destrucción alveolar. Este tipo de DBP es actualmente cada vez menos frecuente. La progresión clínica de alguno de estos pacientes puede ir en franco deterioro de la función pulmonar y de las imágenes radiológicas, llegando a falla respiratoria crónica con hipertensión pulmonar que puede desencadenar en cor pulmonale ${ }^{1,4}$.

\section{Nueva DBP}

Con la introducción de los corticoides antenatales, el uso postnatal de surfactante exógeno y de nuevos modos de asistencia ventilatoria mecánica; junto con estrategias ventilatorias más conservadoras o gentiles, a significado un aumento notorio en la sobrevivencia de los RN prematuros, especialmente de extremo bajo peso (menor de $1.000 \mathrm{~g}$ ) y/o de menos 28-30 semanas de edad gestacional; observándose una nueva presentación de la $\mathrm{DBP}^{3,4}$. Se trata de

\section{Tabla 2. Porcentaje de RN de muy bajo peso sobrevivientes con DBP. Hospital G. Grant Benavente Concepción, Chile}

\begin{tabular}{cccc}
\hline Peso (g) & $\begin{array}{c}\mathbf{O}_{\mathbf{2}} \text { dependencia } \\
\mathbf{2 8} \text { días } \\
\mathbf{\%}\end{array}$ & $\begin{array}{c}\mathbf{O}_{\mathbf{2}} \text { dependencia } \\
\mathbf{3 6} \text { sem } \\
\mathbf{\%}\end{array}$ \\
\hline $500-$ & 749 & 67,4 & 43,0 \\
$750-$ & 999 & 57,3 & 29,9 \\
$1000-1249$ & 22,3 & 12,5 \\
$1250-1499$ & 5,3 & 3,4 \\
\hline
\end{tabular}


neonatos generalmente de extremo bajo peso con dificultad respiratoria inicial leve o ausente, que mejora rápidamente con la administración de surfactante, que luego de algunos días o semanas, con o sin el apoyo de ventilación mecánica, sin oxígeno adicional o con mínimos requerimientos (período de luna de miel), inicia nuevamente dificultad respiratoria y aumento de los requerimientos de oxígeno. Este deterioro puede ser desencadenado por una infección sistémica o pulmonar y/o por la reapertura del ductus arterioso ${ }^{4,13}$. En este tipo de DBP los cambios radiológicos suelen ser sutiles, con leve aumento de la densidad pulmonar o infiltrados intersticiales bilaterales e hiperinsuflación (figura 2). Su evolución es arrastrada o benigna sin cambios significativos entre un día y otro, con requerimientos de oxígeno en general bajos. Lo que caracteriza a esta nueva DBP es la detención del desarrollo pulmonar con detención de la septación alveolar, dando como resultado alvéolos más grandes y en menor cantidad $^{5}$. También se ha observado una detención en el desarrollo vascular, con menos capilares pulmonares y con menor compromiso de las

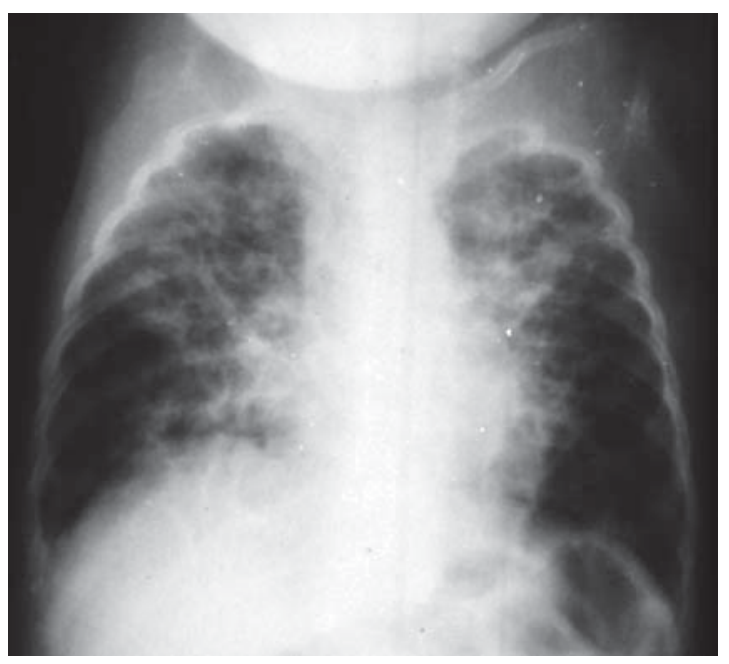

Figura 1. Radiografía de tórax de $\mathrm{RN}$ prematuro de 26 semanas PN (660 gr) con DBP severa a los 4 meses de edad, dependiente de oxígeno adicional. Se visualizan imágenes quísticas, con zonas de hiperinsuflación pulmonar y líneas gruesas intersticiales bilaterales que corresponden a fibrosis pulmonar (etapa 4 de DBP según clasificación de Northway) ("DBP clásica severa"). vías aéreas ${ }^{4,5}$. Luego de producido el daño pulmonar algunos de estos niños pueden requerir ventilación mecánica y aumento de las concentraciones de oxígeno por varias semanas o meses $^{4,13}$. Las diferencias entre la DBP clásica y la nueva DBP se visualizan en la tabla 3.

\section{Patogénesis}

La etiopatogenia de la DBP es claramente multifactorial; y los primeros factores de riesgo reportados por Northway hace 40 años se mantienen en plena vigencia. Es así como la prematuridad, el síndrome de dificultad respiratoria; las altas concentraciones de oxígeno y la injuria producida por la ventilación mecánica siguen teniendo un rol fundamental en el desarrollo de la $\mathrm{DBP}^{1,4,13}$. Paralelamente en los últimos años se han ido agregando otros factores involucrados en su patogenia como son: la inflamación y/o infección, la administración excesiva de líquidos, el edema pulmonar, la persistencia o reapertura del ductus arterioso, el aumento de la resistencia de las vías aéreas, inmadurez de los sistemas antioxidantes, deficiencias nutricionales, insuficiencia adrenal precoz, y predisposición genética ${ }^{4,15-17}$ (figura 3).

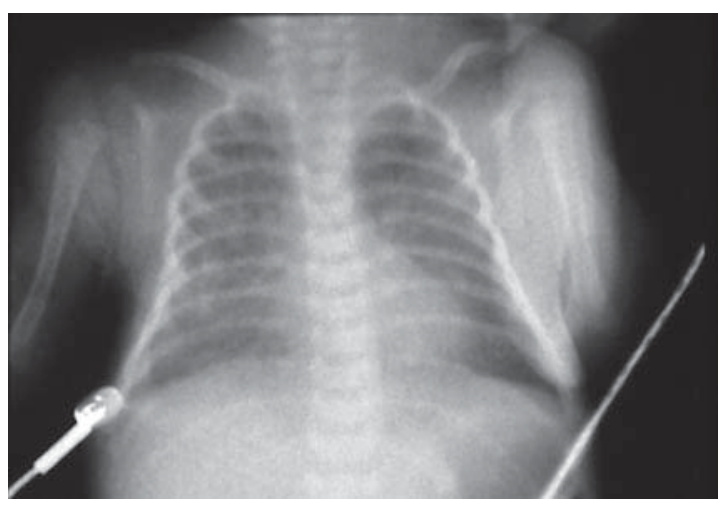

Figura 2. Radiografía de tórax de $\mathrm{RN}$ pretérmino de 27 semanas de edad gestacional, PN $910 \mathrm{~g}$ con DBP leve a los 30 días de vida. Se visualizan campos pulmonares sólo con discreta opacidad pulmonar. ("Nueva DBP”). 
Tabla 3. Diferencias entre DBP Clásica y Nueva DBP

\begin{tabular}{|c|c|c|}
\hline Características & DBP Clásica & Nueva DBP \\
\hline Origen & Descrito por Northway en 1967 & Descrito por Jobe en 1999 \\
\hline Peso Nac. (g) & $500-1800$ & $<1250$ \\
\hline SDR inicial & Severo & Leve o moderado \\
\hline Oxígeno suplementario & Elevado & Bajo \\
\hline $\begin{array}{l}\text { Requerimiento } \\
\text { ventilación mecánica }\end{array}$ & $\begin{array}{l}\text { Habitualmente por insuficiencia respiratoria } \\
\text { severa }\end{array}$ & $\begin{array}{l}\text { Habitualmente por apnea o falta de esfuerzo } \\
\text { respiratorio (inmadurez pulmonar) }\end{array}$ \\
\hline $\begin{array}{l}\text { Tipo ventilación } \\
\text { mecánica }\end{array}$ & $\begin{array}{l}\text { Agresiva: volúmenes corrientes elevados } \\
\text { (volutrauma) }\end{array}$ & $\begin{array}{l}\text { Conservadora o gentil, pero prolongada } \\
\text { (volúmenes corrientes pequeños) }\end{array}$ \\
\hline Cambios histológicos & $\begin{array}{l}\text { Lesiones severas de vías aéreas, hiperplasia, } \\
\text { metaplasia y fibrosis pulmonar }\end{array}$ & $\begin{array}{l}\text { Vías aéreas con menos hiperplasia y nula o } \\
\text { leve fibrosis pulmonar }\end{array}$ \\
\hline Alvéolos & En cantidad y tamaño relativamente normal & $\begin{array}{l}\text { Alvéolos en cantidad disminuida y de mayor } \\
\text { tamaño (detención desarrollo pulmonar) }\end{array}$ \\
\hline Capilares pulmonares & $\begin{array}{l}\text { En número normal, pero con lesiones vasculares } \\
\text { diversas }\end{array}$ & Disminuidos, con dismorfia \\
\hline Hipertensión pulmonar & Frecuente & Infrecuente \\
\hline Factores de riesgo & $\begin{array}{l}\text { Principalmente SDR severo, volutrauma y } \\
\text { elevadas concentraciones de } \mathrm{O}_{2}\end{array}$ & $\begin{array}{l}\text { Infección, ductus persistente, déficit enzimá- } \\
\text { ticos, déficit nutricionales }\end{array}$ \\
\hline Mortalidad & Incierta & Baja \\
\hline Pronóstico & Regular & En general bueno \\
\hline
\end{tabular}

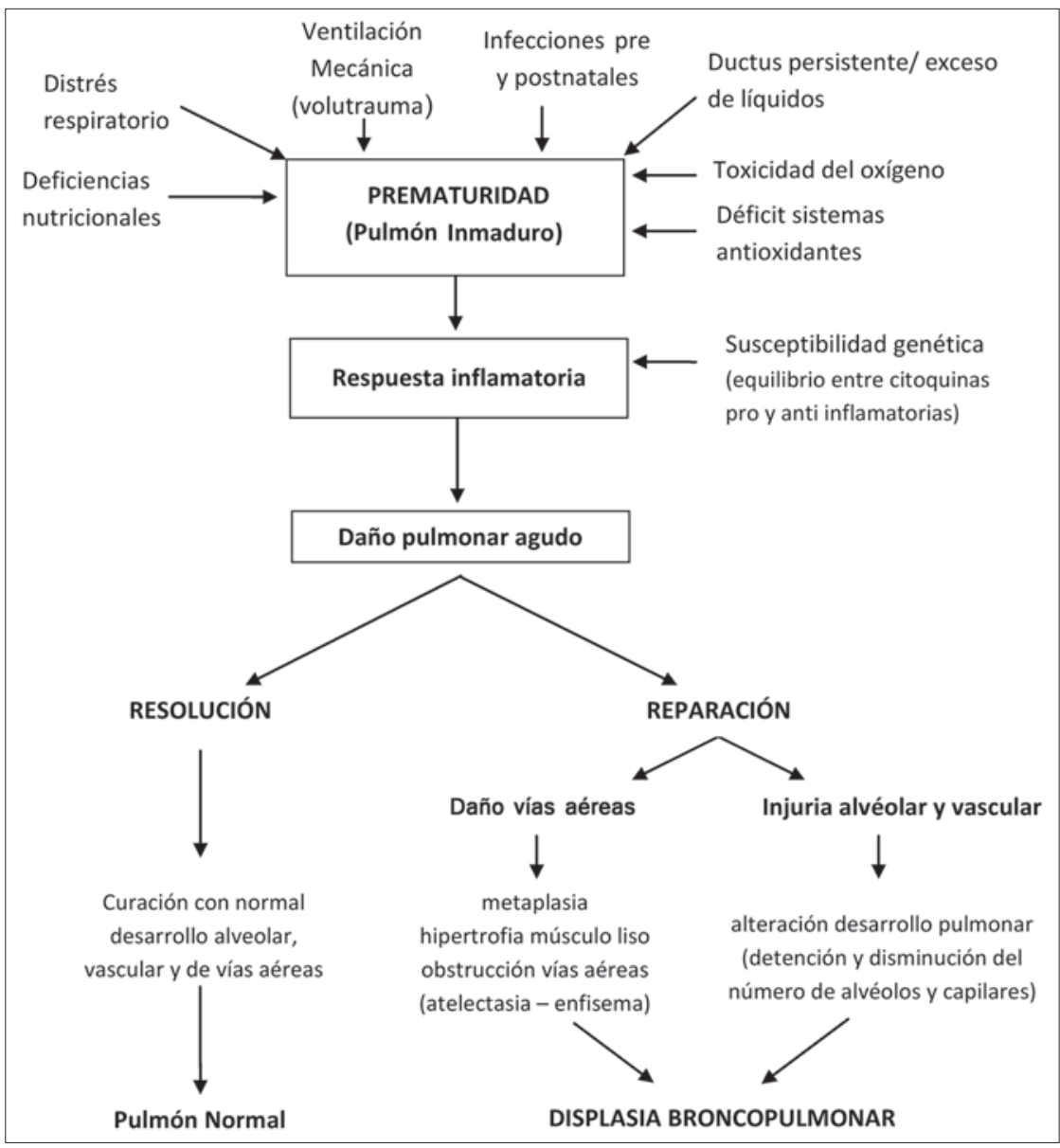

Figura 3. Patogénesis displasia broncopulmonar. 


\section{Prematuridad-Inmadurez y Desarrollo Pulmonar}

La incidencia de DBP es mayor mientras menor es la edad gestacional y el peso de nacimiento del RN. Actualmente es muy raro que un $\mathrm{RN}$ mayor de 32 semanas de edad gestacional desarrolle DBP ya que esta patología esta concentrada fundamentalmente en los RN muy inmaduros o de extremo bajo peso $(<1000 \mathrm{~g})$ y de menos 28 semanas de edad gestacional $^{4,13}$. La mayor vulnerabilidad de estos niños estaría dada por un desarrollo incompleto del pulmón y por déficit o ausencia de diversos factores que pueden proteger al pulmón inmaduro que está en vías de desarro$10^{4,15}$. Se sabe que a las 24 semanas de gestación el pulmón está en etapa canalicular del desarrollo, progresando a etapa sacular alrededor de las 30 semanas $^{13,15}$. Por tanto, el pulmón muy inmaduro puede ser fácilmente dañado por elevadas concentraciones de oxígeno y por el trauma producido por la ventilación mecánica artificial que habitualmente es necesario para la sobrevivencia de estos niños muy inmaduros ${ }^{16,18}$.

Esto explica en parte que la denominada nueva DBP presente menor cantidad de alvéolos y de mayor tamaño, concordantes con una detención o alteración del desarrollo pulmonar normal ${ }^{2,4,15,16}$. Además el factor de crecimiento endotelial vascular también estaría alterado, comprometiendo la vascularización, y por ende la alveolarización del pulmón ${ }^{15,16}$. Esta disminución en el número de alvéolos y capilares pulmonares disminuye la superficie de intercambio gaseoso, siendo necesario aumentar el aporte de oxígeno adicional para mantener una oxigenación normal ${ }^{19,20}$.

\section{Oxígenoterapia y Toxicidad}

Desde la descripción inicial de la DBP se ha demostrado el efecto tóxico del oxígeno a nivel pulmonar; por este motivo debe considerarse como una droga y como tal, debe estar indicada, dosificada y controlada tratando de administrarse durante el menor tiempo posible. Aunque la causa de la DBP no se conoce, existe una fuerte asociación clínica y experimental entre este trastorno y la exposición a elevadas concentraciones de oxígeno ${ }^{1,21}$. El pulmón al recibir las altas concentraciones de oxígeno es uno de los órganos más afectados, pudiendo haber alteraciones en la permeabilidad capilar, con transudados a los alvéolos, necrosis de células alveolares tipo 1, hiperplasia escamosa epitelial, atelectasia, y hemorragia intersticial y alveolar ${ }^{19,20}$. Estos efectos se deberían a la formación de radicales libres derivados del oxígeno, que producirían un stress oxidativo con alteración de la integridad de las membranas y daño estructural intracelular ${ }^{19}$. El efecto de la hiperoxia a través de los radicales libres en los $\mathrm{RN}$ prematuros se ve favorecida por la disminución de mecanismos antioxidantes en este tipo de niños ${ }^{22}$.

La concentración de oxígeno que es tóxica para el pulmón depende de un gran número de variables, incluyendo el nivel de madurez del pulmón, el estado nutricional del paciente, el porcentaje y duración de la exposición a oxígeno adicional, a la cantidad de antioxidantes presentes en el tejido pulmonar, etc. Hasta el momento no se han establecido niveles seguros de oxígeno inspirado, ni el tiempo de duración de este, por lo tanto, cualquier concentración mayor que la que existe en el aire ambiental, puede aumentar el riesgo de daño pulmonar, si se administra por períodos prolongados. Un ensayo clínico que evaluó el desarrollo pondoestatural y neurosensorial a los 12 meses de edad corregida con dos diferentes niveles de saturometría, una estándar entre 91 y 94\% y otra elevada entre 95 y $98 \%$, no mostró diferencias entre los dos grupos de niños en relación al peso, talla o circunferencia craneana, ni tampoco en el desarrollo neurosensorial. No obstante en aquellos RN asignados al nivel alto de saturometría (95-98\%) se detectó un peor pronóstico pulmonar, falleciendo 6 niños en comparación con sólo uno del grupo con saturometría estandar ${ }^{23}$. Similar situación fue reportada en el estudio de STOP-ROP (Suplemental Therapeutic Oxygen for Prethreshold Retinopathy of Prematurity) ${ }^{24}$, en que el grupo de niños randomizados a niveles altos de saturometrías (96 a 99\%) presentó significativamente mayor número de complicaciones respiratorias como neumonía y/o exacerbaciones de la DBP, en relación al grupo con saturaciones menores, entre $89-94 \%$ (13,2\% vs 8,2\%). Ambos estudios revelan claramente que mantener elevadas saturaciones de oxíge- 
no es dañino para el pulmón y por ende favorecerían el desarrollo de DBP. Sin embargo, hasta hoy se desconoce cual sería la saturometría de oxígeno ideal para mantener a un RN, especialmente aquellos de extremo bajo peso y durante las primeras semanas de vida; pero hay consenso mayoritario que saturaciones de oxígeno sobre $95 \%$ producen más daño pulmo$\operatorname{nar}^{23,24}$. Es muy posible que en la etapa aguda del distrés respiratorio saturaciones entre 88 y $95 \%$ sean adecuadas y producirían menor daño pulmonar.

\section{Ventilación Mecánica}

El término de DBP se introdujo después del inicio de la ventilación mecánica en el RN; por lo cual se puede inferir que es un factor de riesgo primordial en el desarrollo de la DBP. Por otra parte, raramente se aprecia esta afección en $\mathrm{RN}$ que no han requerido nunca soporte ventilatorio mecánico ${ }^{4}$. Lo más dañino de la ventilación mecánica es el exceso de volumen en cada ciclo respiratorio mecánico, lo que se denomina volutrauma, que produce una sobredistensión de la membrana alvéolocapilar, desencadenando una reacción inflamatoria de ésta, con edema intersticial e inactivación del surfactante ${ }^{16,21}$. Paralelamente esta sobredistensión puede producir ruptura alveolar: neumotórax o enfisema intersticial, que son factores que agraven el cuadro de insuficiencia respiratoria y predisponen al $\mathrm{RN}$ a desarrollar $\mathrm{DBP}^{21}$. $\mathrm{Al}$ usar presiones inspiratorias elevadas, también se pueden generar volúmenes corrientes elevados que producen daño pulmonar. Por otra parte, el no usar una presión espiratoria al final de la espiración (PEEP) o ser esta muy baja, se favorece el colapso alveolar, debiendo en cada ciclo inspiratorio despegarse repetidamente las paredes alveolares predisponiendo también a daño de la membrana alveolocapilar, lo que se denomina atelectrauma ${ }^{16,21}$. Además el empleo de altas concentraciones de oxígeno asociado a la ventilación mecánica desencadena un proceso inflamatorio, que predispone a injuria pulmonar $^{21}$. Este fenómeno se ha denominado biotrauma ${ }^{21}$.

Otro factor de importancia en el daño por la ventilación mecánica, está dado por la necesidad de utilizar un tubo endotraqueal, que au- menta la posibilidad de infección pulmonar, dificulta la movilización de secreciones por interferir el normal transporte ciliar, pudiendo este efecto acentuarse si el gas inspirado no esta adecuadamente calentado y humidificado ${ }^{21}$. Cabe recordar que muchas veces se daña la mucosa traqueal y bronquial por un inadecuado y/o excesivo uso de succiones o aspiraciones endotraqueales, facilitando también la colonización y/o infección de las vías aéreas y del parénquima pulmonar ${ }^{25}$.

\section{Infección e Inflamación}

La inflamación del pulmón es uno de los principales factores de riesgos involucrados en la patogénesis de la DBP; habiéndose demostrado una clara asociación entre infección prenatal y postnatal y el desarrollo de $\mathrm{DBP}^{4,26}$. Estudios clínicos en RN prematuros de madres con corioammionitis tienen mayor susceptibilidad de desarrollar DBP, a pesar que esta infección acelera la madurez pulmonar ${ }^{27,28,29}$. Niveles elevados de diversas citoquinas proinflamatorias como son IL-6, IL-8, IL1 $\beta$, TNF- $\alpha$, en el líquido amniótico antes del parto prematuro se han asociado con mayor riesgo de $\mathrm{DBP}^{28}$. Existe suficiente evidencia científica, que demuestra que la inflamación del pulmón, es uno de los principales factores de riesgo para el desarrollo de la $\mathrm{DBP}^{26,30}$. El factor de necrosis tumoral y la IL-6 inducen la producción de fibroblastos y colágeno que están presentes en los niños con $\mathrm{DBP}^{26,30}$. También se han encontrado niveles elevados de leucotrienos en pulmón de niños que desarrollan esta patología y que permanecen elevados hasta los 6 meses de edad $^{31}$. Los leucotrienos causan una serie de alteraciones pulmonares como edema intersticial, producción de mucus y especialmente broncoconstricción y vasoconstricción ${ }^{31}$. Sin embargo, aún se desconoce cual es el factor que inicia y mantiene la respuesta inflamatoria que termina dañando el pulmón.

La inflamación puede ser desencadenada por factores infecciosos: bacterianos, virales o fúngicos ${ }^{26} \mathrm{y}$ diversos factores no infecciosos entre los cuales están los radicales libres de oxígeno, el volutrauma producido por la ventilación mecánica, y el aumento del flujo pulmonar por un ductus arterioso persistente ${ }^{4,15,30}$. Uno 
de los agentes infecciosos que frecuentemente contaminan el líquido amniótico desencadenando una respuesta inflamatoria fetal y neonatal es el mycoplasma Ureaplasma Urealyticum ${ }^{32}$, el cual ha sido aislado del pulmón de niños que desarrollaron $\mathrm{DBP}^{32}$. También se ha demostrado una clara asociación entre infecciones intrahospitalarias sistémicas y una mayor incidencia de $\mathrm{DBP}^{4,13}$, siendo esta aún mayor cuando la infección ocurre simultáneamente con un ductus arterioso persistente ${ }^{33}$. Paralelamente se ha reportado un aumento significativo de células inflamatorias, eicosanoides y diversas citoquinas en las vías aéreas de niños que desarrollan $\mathrm{DBP}^{34}$.

\section{Ductus Arterioso Persistente y Administración de Líquidos}

Se ha reportado que recién nacidos con Síndrome de dificultad respiratoria que reciben exceso de líquido, o no tienen una adecuada diuresis en los primeros días de vida, tienen una mayor incidencia de $\mathrm{DBP}^{35}$. Este exceso de líquido favorece la mantención o reapertura del ductus, el cual produce un aumento del flujo hacia los pulmones, predisponiendo a un edema pulmonar, con deterioro en la mecánica respiratoria ${ }^{36}$, prolongando de esta forma la necesidad de oxígeno suplementario y/o ventilación mecánica. Esta secuencia de eventos puede favorecer el desarrollo de DBP, especialmente si se mantienen por tiempo prolongado. Se ha reportado que mientras mayor es la duración del ductus, mayor es el riesgo de $\mathrm{DBP}^{37}$.

También se ha demostrado que las infecciones nosocomiales producen liberación de citoquinas, que favorecen la reapertura del ductus, o bien que impiden una respuesta satisfactoria al cierre farmacológico de este, favoreciendo por ende su perpetuación, con el posible daño pulmonar ${ }^{15,33}$. Se ha observado una fuerte asociación entre infección sistémica, ductus y $\mathrm{DBP}^{13,33}$.

\section{Deficiencias Nutricionales y Antioxidantes}

El RN de pretérmino de muy bajo peso tiene un mayor gasto energético, que puede llevarlo a un estado catabólico, el cual puede exacerbar el daño pulmonar producido por diferentes noxas. Por lo tanto, tiene gran importancia pre- ocuparse de la nutrición del prematuro extremo desde el nacimiento. Se ha relacionado el déficit de diversos nutrientes, como algunos aminoácidos y algunos elementos trazas como cobre, hierro, zinc, selenio y magnesio en la patogénesis de la $\mathrm{DBP}^{4,38}$. También hay clara evidencia de mayor riesgo de esta patología en deficiencia en vitamina $A^{39}$. Se ha demostrado que la administración de vitamina A en el primer mes de vida tiene un efecto protector contra el desarrollo de DBP, disminuyendo significativamente la incidencia de esta afección $^{39,40}$. El metaanálisis de 7 estudios randomizados mostró que la suplementación sistémica con vitamina A en cantidad suficiente para lograr concentraciones normales de retinol en suero, reduce la dependencia de oxígeno a las 36 semanas postconcepcionales ${ }^{40}$. Además el déficit de vitamina $\mathrm{A}$ en animales de experimentación, muestra alteraciones en las vías aéreas, como pérdida del epitelio ciliar y metaplasia escamosa, que son muy similares a los cambios histológicos observados en niños con $\mathrm{DBP}^{41}$. Paralelamente se ha comprobado que los prematuros tienen una menor concentración de enzimas antioxidantes ${ }^{22}$, las cuales tienen gran importancia en evitar o disminuir el estrés oxidativo producido por los radicales libres de oxígeno ${ }^{22}$. Se ha demostrado una disminución de la enzima antioxidante glutatión, por deficiencia de selenio, al ser este un elemento esencial en su composición ${ }^{42}$. También se ha reportado déficit de la enzima superóxidodismutasa y de la catalasa ${ }^{22}$.

Estos déficit nutricionales y de antioxidantes en los prematuros, podrían en parte ser minimizados con la ingesta exclusiva de leche materna durante las primeras semanas de vida. Recientemente en un estudio prospectivo del Instituto Nacional de Salud de los EE.UU. en más de 1000 recién nacidos de extremo bajo peso, se reportó que la incidencia de DBP fue significativamente menor en los prematuros alimentados con leche materna exclusiva durante los primeros 28 días de vida, comparados con aquellos alimentados con leche artificial en igual período de tiempo ${ }^{43}$. Estos resultados posiblemente se deban a las cualidades inmunológicas y a las altas concentraciones de antioxidantes presentes en la leche materna ${ }^{43}$. 


\section{Aumento de Resistencia de Vías Aéreas}

Se ha demostrado aumento de la resistencia de vías aéreas desde la primera semana de vida, en neonatos que posteriormente desarrollan $\mathrm{DBP}^{44}$, planteando la posibilidad de que la obstrucción de las vías aéreas puede ser secundaria a la hiperplasia, y metaplasia bronquiolar; como también al edema de la mucosa, resultante del trauma por la toxicidad del oxígeno y de la infección ${ }^{4,15}$. Niños con DBP a los 28 días de vida presentan una resistencia pulmonar inspiratoria y expiratoria aumentada, con severa limitación del flujo, especialmente con bajos volúmenes pulmonares ${ }^{45}$. Además en las etapas iniciales de esta patología la capacidad funcional residual esta a menudo disminuida, debido a las atelectasias, pero durante las etapas más tardías existe atrapamiento de gas con hiperinsuflación ${ }^{45}$. Paralelamente el ductus arterioso persistente y la administración excesiva de líquidos, pueden conducir a edema intersticial pulmonar, disminuyendo la distensibilidad pulmonar y aumentando la resistencia de las vías aéreas ${ }^{36}$. También se ha reportado un aumento de la resistencia de las vías aéreas secundaria a la liberación de mediadores de inflamación, como leucotrienios y factor activador de plaquetas en niños con $\mathrm{DBP}^{29}$. Estos cambios en la mecánica pulmonar en los niños con DBP pueden ser evaluados y efectuarse su seguimiento a través de las pruebas de función pulmonar $^{46}$.

\section{Factores Genéticos}

La variabilidad observada en la incidencia y severidad de la DBP entre prematuros con similares factores de riesgos, sugiere que factores genéticos jugarían un importante rol en la patogénesis de esta enfermedad. Recientemente se ha observado una marcada interacción entre los factores genéticos y los factores de riesgo del medio ambiente, en el desarrollo de la $\mathrm{DBP}^{47,48}$. Diversos estudios en mellizos ${ }^{49,50}$ han demostrado que factores genéticos aumentarían la susceptibilidad de la DBP, al encontrarse una mayor asociación o concordancia de esta enfermedad en mellizos monozigóticos en comparación con los dizigóticos; independientemente de la edad gestacional, del peso de nacimiento, género, severidad del distrés respi- ratorio, corticoides antenatales, presencia de ductus, infecciones y de otros factores. $\mathrm{La}$ concordancia de la DBP en los mellizos monozigóticos es aproximadamente 2 veces con respecto a los dizigóticos ${ }^{50}$. El estudio de Lavoie y $\operatorname{cols}^{50}$, demostró que en mellizos monozigóticos de 30 semanas o menos de edad gestacional habría una susceptibilidad genética en el desarrollo de la DBP, definida según el último consenso de esta enfermedad ${ }^{5}$. Dicho estudio encontró también una predisposición genética en la persistencia del ductus arterioso, el cual es uno de los factores de riesgo de $\mathrm{DBP}^{5,37,50}$.

La mayor frecuencia de DBP en el sexo masculino sería otra señal que factores genéticos estarían involucrados en la patogenia de la $\mathrm{DBP}^{11,51}$. La forma por la cual el género masculino predispondría al desarrollo de DBP aún no esta claramente precisada. Se especula que la producción de proteínas, podría estar genéticamente determinada, produciéndose diferencias en las respuestas de las citoquinas pro y antiinflamatorias entre el sexo masculino y femenino. De igual forma, se ha publicado que la raza blanca también se ha asociado con un aumento del riesgo de esta enfermedad, luego de efectuar los ajustes pertinentes por otros factores de riesgo ${ }^{52}$.

Numerosos genes son requeridos para el normal crecimiento y desarrollo del pulmón, los cuales probablemente contienen secuencia de variaciones que modularían el riesgo de DBP. Diversos estudios han identificado potenciales genes candidatos, especialmente con respecto a las proteínas que forman el surfactante pulmonar y de algunas citoquinas ${ }^{53,54}$. Se ha reportado que variaciones genéticas en los genes de las proteínas que forman el surfactante pulmonar, influyen en la severidad del distrés respiratorio y el subsecuente desarrollo de $\mathrm{DBP}^{55}$.

Los últimos estudios efectuados en mellizos representan una expansión en el conocimiento de la patogénesis de la DBP, en el sentido que esta enfermedad no sólo se debería a factores ambientales, si no que, también sería una consecuencia de la interacción entre diferentes genes y el medio ambiente. El desafío actual es tratar de identificar los posibles genes que contribuirían al desarrollo de la DBP y determinar la interacción entre esos genes y los estímulos 
ambientales específicos, que adversamente afectan el proceso de reparación normal del pulmón inmaduro que ha sido injuriado.

\section{Conclusión}

La DBP luego de 40 años, continúa siendo la enfermedad pulmonar crónica más frecuente que afecta a los RN prematuros de muy bajo peso. En estas 4 décadas se han producido cambios en la definición; epidemiología, hallazgos radiológicos y patogénesis. La DBP clásica descrita por Northway y cols, en 1967 ha cambiado a formas más leves de daño pulmonar crónico, la denominada "nueva DBP", caracterizada por un deterioro en el crecimiento postnatal del pulmón. Estas dos formas de enfermedad pulmonar crónica, difieren en su presentación, mecanismos patogénicos y consecuencias a largo plazo.

En los últimos años, a los factores de riesgo ya establecidos, se han agregados otros, como la inflamación-infección, déficit de antioxidantes, la presencia de ductus arterioso persistente, y factores genéticos.

\section{Referencias}

1.- Northway WH Jr, Rosan RC, Porter DY: Pulmonary disease following respirator therapy of hyaline membrane disease: bronchopulmonary dysplasia. N Engl J Med 1967; 276: 357-68.

2.- Jobe AH: The new BPD: an arrest of lung development. Pediatr Res 1999; 46: 641-3.

3.- Fanaroff AA, Stoll BJ, Wright LL, et al: Trends in neonatal morbidity and mortality for very low birthweight infants. Am J Obstet Gynecol 2007; 196: 147.e1-147.e8.

4.- Bancalari E, Claure N, Sosenko IRS: Bronchopulmonary dysplasia: changes in pathogenesis, epidemiology and definition. Semin Neonatol 2003; 8: 63-71.

5.- Jobe AH, Bancalari E: Bronchopulmonary Dysplasia. Am J Respir Crit Care Med 2001; 163: 1723-9.

6.- Bancalari E, Abdenour G, Feller R, Gannon J: Bronchopulmonary dysplasia: Clinical presentation. J Pediatrics 1979; 95: 819-23.

7.- Shennan A, Dunn M, Ohlsson A, et al: Abnormal Pulmonary Outcomes in Premature Infants: Prediction From Oxygen Requirement in the Neonatal Period. Pediatrics 1988; 82: 527-32.

8.- Bhandari A, Panitch HB: Pulmonary Outcomes in
Bronchopulmonary Dysplasia. Semin Perinatol 2006; 30: 219-26.

9.- Anderson P, Doyle LW: Neurodevelopmental Outcome of Bronchopulmonary Dysplasia. Semin Perinatol 2006; 30: 227-32.

10.- Walsh M, Yao Q, Gettner P, et al: Impact of a Physiologic Definition on Bronchopulmonary Dysplasia Rates. Pediatrics 2004; 114: 1305-11.

11.- Tapia J, Agost D, Alegría A, et al: Bronchopulmonary dysplasia: incidence, risk factors and resource utilization in a population of South American very low birth weight infants. J Pediatr (Rio J) 2006; 82: 15-20.

12.- Lemons A, Bauer $C R$, Oh W, et al: Very low birth weight outcomes of the National Institue of Child Health and Human Development neonatal Research Network, January 1995 through December 1996. Pediatrics 2001; 107: 1-8.

13.- Rojas M, González A, Bancalari E, et al: Changing trends in the epidemiology and pathogenesis of chronic lung disease. J Pediatr 1995; 126: 605-10.

14.- Ehrenkranz RA, Walsh MC, Vohr BR, et al: Validation of the National Institutes of Health consensus definition of bronchopulmonary dysplasia. Pediatrics 2005; 116: $1353-60$.

15.- Sosenko I, Bancalari E: New Developments in the Presentation, Pathogenesis, Epidemiology and Prevention of Bronchopulmonary Dysplasia. In: Polin R, Bancalari E, (editors) The Newborn Lung. Neonatology Question and Controversies. Saunders Elsevier, Philadelphia, 2008: 187-207.

16.- Kinsella JO, Greenough A, Abman SH: Bronchopulmonary Dysplasia. Lancet 2006; 367: 1421-31.

17.- $H u$ C, Bancalari A: Incidencia y Factores Asociados a Displasia Broncopulmonar. Rev Chil Pediatr 2005; 76 : $662 \mathrm{~A}$.

18.- Bancalari E, González A: Clinical course and lung function abnormalities during development of neonatal chronic lung disease. In: Bland RD, Coalson JJ editors. Chronic lung disease in early infancy. New York: Marcel Dekker; 2000. p. 41-64.

19.- Husain AN, Siddiqui NH, Stocker JT: Pathology of arrested acinar development in postsurfactant bronchopulmonary dysplasia. Human Pathol 1998; 29: 710-7.

20.- Coalson JJ: Pathology of chronic lung disease of early infancy. In: Bland RJ, Coalson JJ, eds. Chronic lung disease in early infancy. New York: Marcel Dekker; 2003: 85-124.

21.- Clark R, Gerstmann D, Jobe A, et al: Lung injury in neonates: Causes, strategies for prevention, and longterm consequences. J Pediatr 2001; 139: 478-86.

22.- Asikainen TM, Raivio KO, Saksela M, Kinnula VL: Expression and developmental profile of antioxidant enzymes in human lung and liver. Am J Respir Cell Mol Biol 1998; 19: 942-9.

23.- Askie LM, Henderson-Smart DJ, Irwig L, Simpson JM: Oxygen-saturation targets and outcomes in extremely preterm infants. N Engl J Med 2003; 349: 959-67.

24.- STOP-ROP Multicenter Study Group: Supplemental therapeutic oxygen for prethreshold retinopathy of 
prematurity (STOP-ROP), a randomized controlled trial. I: Primary outcomes. Pediatrics 2000; 105: 295310.

25.- Bailey C, Kattwinkel J, Teja K, Buckley T: Shallow Versus Deep Endotracheal Suctioning in Young Rabbits: Pathologic Effects on the Tracheobronchial Wall. Pediatrics 1988; 82: 746-51.

26.- Speer $C$ : Inflamation and bronchopulmonary dysplasia. Seminars in Neonatology 2003; 8: 29-38.

27.- Watterberg KL, Demers LM, Scott SM, Murphy S: Chorioamnionitis and early lung inflammation in infants in whom bronchopulmonary dysplasia develops. Pediatrics 1996; 97: 210-5.

28.- Yoon BH, Romero R, Jun JK, et al: Amniotic fluid cytokines (interleukin-6, tumor necrosis factor-a, interleukin-lâ, and interleukin-8) and the risk for the development of bronchopulmonary dysplasia. Am J Obstet Gynecol 1997; 177: 825-30.

29.- Groneck $P$, Speer $C P$ : Inflamatory mediators and bronchopulmonary dysplasia. Arch Dis Child 1995; 73: F1-F3.

30.- Pierce MR, Bancalari E: The role of inflammation in the pathogenesis of bronchopulmonary dysplasia. Pediatr Pulmonol 1995; 19: 371-8.

31.- Cook AJ, Yuksel B, Sampson AP, et al: Cysteinyl leukotriene involvement in chronic lung disease in premature infants. Eur Respir J 1996; 9: 1907-12.

32.- Viscardi R, Manimtim W, Sun C, et al: Lung Pathology in Premature Infants with Ureaplasma urealyticum Infection. Pediatric and Developmental 2002; 5: 14150

33.- González A, Sosenko IRS, Chandar J, et al: Influence of infection on patent ductus arteriosus and chronic lung disease in premature infants weighing 1000 grams or less. J Pediatr 1996; 128: 470-8.

34.- Groneck P, Gotze-Speer B, Oppermann M, et al: Association of pulmonary inflammation and increased microvascular permeability during the development of bronchopulmonary dysplasia: a sequential analysis of inflammatory fluids of high-risk preterm neonates. Pediatrics 1994; 93: 712-8.

35.- Oh W, Poindexter BB, Perritt R, et al: Association between fluid intake and weight loss during the first ten days of life and risk of bronchopulmonary dysplasia in extremely low birth weight infants. J Pediatr 2005; 147: 786-90.

36.- Gerhardt T, Bancalari E: Lung compliance in newborns with patent ductus arteriosus before and after surgical ligation. Biol Neonate 1980; 38: 96-105.

37.- Bancalari E, Claure N, González A: Patent ductus arteriosus and respiratory outcome in premature infants. Biol Neonate 2005; 88: 192-201.

38.- Cadwell JL: Evidence for magnesium deficiency in the pathogenesis of bronchopulmonary dysplasia. Magnesium Res 1996; 9: 205-16.

39.- Tyson JE, Wright LL, Oh $Q$, et al: Vitamin A supplementation for extremely low birth weight infants. N Engl J Med 1999; 340: 1962-8.

40.- Darlow BA, Graham PJ: Vitamin A supplementation for preventing morbidity and mortality in very low birthweight infants. Cochrane Database Syst Rev 2002; 4: CD000501.

41.- Takahashi Y, Miura T, Takahashi K: Vitamin A is involved in maintenance of epithelial cells on the bronchioles and cells in the alveoli of rats. J Nutr 1993; 123: 634-641.

42.- Falciglia HS, Johnson JR, Sullivan J, et al: Role of antioxidant nutrients and lipid peroxidation in premature infants with respiratory distree syndrome and bronchopulmonary dysplasia. Am J Perinatol 2003; 20: 97-107.

43.- Duara $S$, Poindexter B, Saha $S$, et al: Human milk as protection against bronchopulmonary dysplasia (BPD) in extremely low birth weight infants. Pediatr Res 2004; 55: 506A.

44.- Goldman SL, Gerhardt T, Sonni R, et al: Early prediction of chronic lung disease by pulmonary function testing. J Pediatr 1983; 120: 613-7.

45.- Wolfson MR, Bhutani BK, Shaffer TH, Bowen FW Jr: Mechanics and energetics of brething helium in infants with bronchopulmonary dysplasia. J Pediatr 1984; 104 : 752-7.

46.- Gerhardt T, Hehre D, Feller R, Reinfenberg L, Bancalari E: Serial determination of pulmonary function in infants with chronic lung disease. J Pediatr 1987; 110: 448-56.

47.- Hallman $M$, Haataja $R$ : Genetic influences and neonatal lung disease. Semin Neonatol 2003; 8 (1) 19-27.

48.- Bhandari V, Gruen JR: Genetics of bronchopulmonary dysplasia. Semin Perinatol 2006; 30 (4): 185-91.

49.- Parker RA, Lindstrom DP, Cotton RB: Evidence from twin study implies possible genetic susceptibility to bronchopulmonary dysplasia. Semin Perinatol 1996; 20 (3): 206-9.

50.- Lavoie PM, Phan C, Kang KL: Heritability of bronchopulmonary dysplasia defined per the consensus statement of the National Institutes of Health. Pediatrics 2008; 122 (3): 479-85.

51.- Henderson-Smart DJ, Hutchinson JL, Donoghue DA, et al: Prenatal predictors of chronic lung disease in very preterm infants. Arch Dis Child Fetal Neonatal Ed 2006; 91: F40-5.

52.- Parker RA, Lindstrom DP, Cotton RB: Improved survival accounts for most, but not all, of the increase in bronchopulmonary displasia. Pediatrics 1992; 90: 663-8.

53.- Weber B, Borkhardt A, Stoll-Becker S, Reiss I, Gortner $L$ : Polymorphisms of surfactant protein A genes and the risk of bronchopulmonary dysplasia in preterm infants. Turk J Pediatr 2000; 42 (3): 181-5.

54.- Strassberg SS, Cristea IA, Qian D, Parton LA: Single nucleotide polymorphisms of tumor necrosis factoralpha and the susceptibility to bronchopulmonary dysplasia. Pediatr Pulmonol 2007; 42 (1): 29-36.

55.- Hallman M, Haataja R: Surfactant protein polymorphisms and neonatal lung disease. Semin Perinatol 2006; 30: 350-61. 